\title{
LUPUS NEPHRITIS
}

\section{Multitarget induction therapy for LN}

Multitarget therapy should be considered as an alternative to conventional therapy for induction treatment of lupus nephritis (LN), say the authors of a new study. Zhi-Hong Liu and colleagues found that patients who received multitarget therapy, comprising mycophenolate mofetil, tacrolimus and steroids, were more likely than those who received conventional therapy, comprising intravenous cyclophosphamide and steroids, to achieve complete remission, with similar incidences of adverse events in the two groups.

Given the fundamental role of immune dysregulation in the pathogenesis of LN, Liu and colleagues hypothesized that targeting multiple aspects of the immune response with combined immunosuppressants might improve outcomes for affected patients. An earlier pilot study indicated that a multitarget regimen was more likely than conventional therapy to induce remission in patients with concurrent class IV and V LN.
To further assess the efficacy and safety of this multitarget induction regimen, the researchers performed a randomized open-label trial across 26 renal centres in China.

After 24 weeks of therapy, significantly more patients on multitarget therapy than on conventional therapy achieved complete remission (45.9\% versus $25.6 \%$, $P<0.001$ ). Overall response (complete and partial remission) was also higher in the multitarget therapy group than in the conventional treatment group (83.5\% versus $63.0 \%, P<0.001)$, and the median time to overall response was shorter in the multitarget group ( 8.9 weeks versus 13.0 weeks). On the basis of their findings, the researchers support use of the multitarget approach for induction treatment of LN.

Susan J. Allison

Original article Liu, Z.-H. et al. Multitarget therapy for induction treatment of lupus nephritis. Ann. Intern. Med. doi:10.7326/M14-1030 